\title{
Surface Approximation and Interpolation via Matrix SVD
}

\author{
Andrew E. Long and Clifford A. Long
}

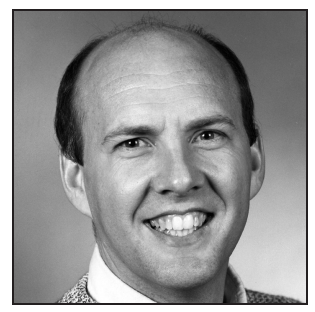

Andy Long (longa@nku.edu) is at Northern Kentucky University. He received his Ph.D. in applied mathematics at the University of Arizona (1994), and has since taught in Wisconsin, been a Fulbright Scholar in Benin, and worked in applications of mathematics to public health. His interests include scientific computing, linear algebra, and mathematical biology.

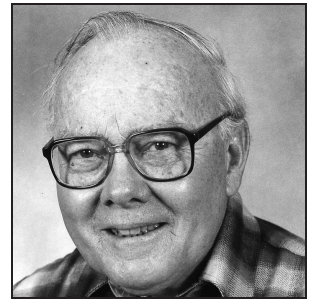

\begin{abstract}
Cliff Long (clong@wcnet.org), a Professor Emeritus in Mathematics and Statistics at Bowling Green State University, received his Ph.D. in 1960 from the University of Illinois. Active in the Ohio Section of the MAA for many years, he continues his interests in the visualization of mathematics by utilizing computer graphics as well as carved wooden sculptures to illustrate related mathematical ideas.
\end{abstract}

Applications of mathematics and statistics often require finding a smooth approximation to a finite set of data points, or interpolating the data points. Mathematicians frequently use cubic spline interpolation when the points lie in a plane, whereby the points are connected with a continuous curve made up of cubic polynomials pieced together in such a way that the combined curve has continuous first and second derivatives at the junction points. There are many other interpolation techniques, such as Lagrange polynomials and Hermite cubics $[2,8]$. Furthermore, when the data points are at all uncertain, smoothing procedures such as a least squares approximation may be preferable to exact interpolation.

Our interest here is in showing how to extend such planar techniques to handle three-dimensional (3D) data points, by using the Singular Value Decomposition (SVD) of a matrix. While there are many procedures for interpolating and approximating 3-D data sets $[4, \mathbf{8}, \mathbf{9}$ ], ours is straightforward and easy to program using Matlab, Mathematica, Maple, Xlispstat or any computer software package that includes an SVD routine. Using any smooth planar data fitting technique, the SVD method yields a smooth surface fit to a given 3D data set. Most other procedures are patch-orientedthe individual surface patches are determined by smoothness conditions imposed along the patch edges. Our surfaces are based directly on the data points and the choice of planar approximation or interpolation technique; no surface patches are explicitly involved.

We first describe and illustrate the method for fitting a surface $z=z(x, y)$ to a simple gridded data set $\left(x_{i}, y_{j}, z_{i j}\right)$, where $z_{i j}=A_{i j}$ for a specific matrix $A$. The process of fitting a surface to a matrix of numbers or points we will call skinning the matrix, even though we are putting a skin on rather than taking one off. 


\section{The SVD technique.}

At the heart of our technique is the general singular value decomposition theorem for matrices. Any $m \times n$ matrix $A$ with real entries can be written as a linear combination

$$
A=\sum_{k=1}^{r} \sigma_{k} u_{k} v_{k}^{T}
$$

where $r$ is the rank of $A$. The $\sigma_{k}$ are positive numbers, ordered from greatest to smallest, called the singular values of $A$; the vectors $u_{k}$ are orthonormal vectors in $\mathrm{R}^{m}$ (called left singular vectors); and the vectors $v_{k}$ are orthonormal vectors in $\mathrm{R}^{n}$ (right singular vectors). Each outer product $u_{k} v_{k}^{T}$ is an $m \times n$ matrix of rank one formed by ordinary matrix multiplication of the column vector $u_{k}$ by the row vector $v_{k}^{T}$. For more information about the SVD, see $[\mathbf{1}, \mathbf{3}, \mathbf{5}, \mathbf{7}]$.

Our idea is simple. Given data points $\left(x_{i}, y_{j}, A_{i j}\right)$, we form the SVD of the $m \times n$ matrix $A=\left[A_{i j}\right]$ and use any chosen planar method to interpolate or approximate the $r$ data sets $\left(x_{i},\left(u_{k}\right)_{i}\right), 1 \leq i \leq m$, associated with the left singular vectors $u_{k}$, by a function $u_{k}(x)$. Similarly we fit a function $v_{k}(y)$ to each of the data sets $\left(y_{j},\left(v_{k}\right)_{j}\right), 1 \leq$ $j \leq n$. Then the function

$$
z(x, y)=\sum_{k=1}^{r} \sigma_{k} u_{k}(x) v_{k}(y)
$$

skins our matrix data set. That is, $z\left(x_{i}, y_{j}\right)=\sum_{k=1}^{r} \sigma_{k} u_{k}\left(x_{i}\right) v_{k}\left(y_{j}\right)$, which is equal to or approximates (depending on whether we interpolated or approximated the planar data) the desired value

$$
A_{i j}=\sum_{k=1}^{r} \sigma_{k} u_{i k} v_{j k} .
$$

The skinning function of (2) is a weighted linear combination of "outer functions", with the singular values as coefficients. This may remind readers of the eigenfunction expansions of solutions to boundary value problems that are encountered in solving partial differential equations by the method of separation of variables.

The differentiability of the interpolating function $z(x, y)$ is an immediate consequence of the differentiability of the singular vector interpolants, as we see from (2). Thus, for example, in order to obtain $C^{2}$ smoothness of the skinning surface, which is typically required for industrial milling processes, we need only use twicedifferentiable interpolants to the planar data sets associated with the singular vectors.

Statisticians sometimes smooth matrix data by dropping from the SVD all terms that correspond to singular values smaller than a given threshold, construing these terms as random measurement errors, or noise. The same procedure can be applied here: one can interpolate the singular vector data sets that correspond to the larger singular values, but drop from (2) the terms that involve singular values smaller than the chosen threshold. The result will be a smoothed surface approximant (rather than interpolant) of the 3-D data set.

Example 1. Consider the problem of skinning the $6 \times 7$ matrix $A$ 


$$
A=\left[\begin{array}{ccccccc}
0.0 & 3.6 & 11.2 & 14.8 & 24.4 & 30.0 & 49.6 \\
0.0 & 7.2 & 22.4 & 29.6 & 48.8 & 60.0 & 99.2 \\
0.0 & 10.8 & 33.6 & 44.4 & 73.2 & 90.0 & 148.8 \\
0.0 & 14.4 & 200.0 & 59.2 & 97.6 & 120.0 & 198.4 \\
0.0 & 18.0 & 56.0 & 74.0 & 122.0 & 150.0 & 248.0 \\
0.0 & 21.6 & 67.2 & 88.8 & 146.4 & 180.0 & 297.6
\end{array}\right]
$$

created as a sum of two outer products (which means that the SVD expansion of (1) has just two terms). Suppose that the grid of points $\left(x_{i}, y_{j}\right)$ on which our interpolating function is to take the values $A_{i j}$ is given by $\left(x_{1}, \ldots, x_{6}\right)=(2,6,8,13,16,26)$ and $\left(y_{1}, \ldots, y_{7}\right)=(0,2,4,6,8,10,12)$.

Solution. Figure 1 shows the resulting skeleton; i.e., the data points $\left(x_{i}, y_{j}, A_{i j}\right)$ connected by straight line segments. The upper row of Figure 2 shows the piecewise linear and Hermite $C^{1}$ interpolants for the left and right singular vector data sets corresponding to the largest singular value of $A$. The lower row shows the outer product of these two vectors and the function interpolant corresponding to the Hermite interpolation scheme, weighted by the singular value.

Hermite $C^{1}$ interpolants are piecewise cubics obtained by assigning slopes at each of the data points. For each subinterval the four conditions (matching data values and slopes at the "nodes") determine a unique cubic for that subinterval. We used the simple derivative approximation $g^{\prime}\left(t_{i}\right)=\frac{g\left(t_{i+1}\right)-g\left(t_{i-1}\right)}{t_{i+1}-t_{i-1}}$ to estimate slopes at the internal nodes.

The top row of Figure 3 similarly shows the second rank-one outer product matrix along with its interpolating Hermite surface; in the bottom row we show the data matrix and the resulting function interpolating surface, formed by summing the rank-one interpolating surfaces.

Example 2. We used SVD interpolation to create a finer resolution "computer bust" of Abraham Lincoln (Figure 4), starting from a scan of a bust using $36 \times 48$ (1728) estimated points. These original points were only accurate to an eighth of an inch in the radial direction: anyone scrutinizing our deluxe scanner (Figure 5) can see why.

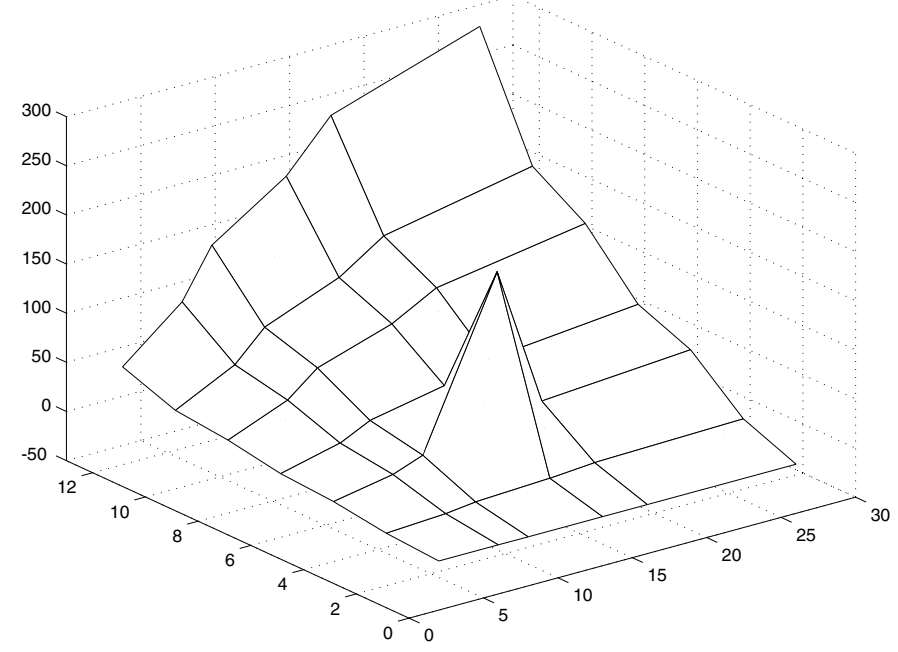

Figure 1. The skeleton of the data matrix $A$ (3) 

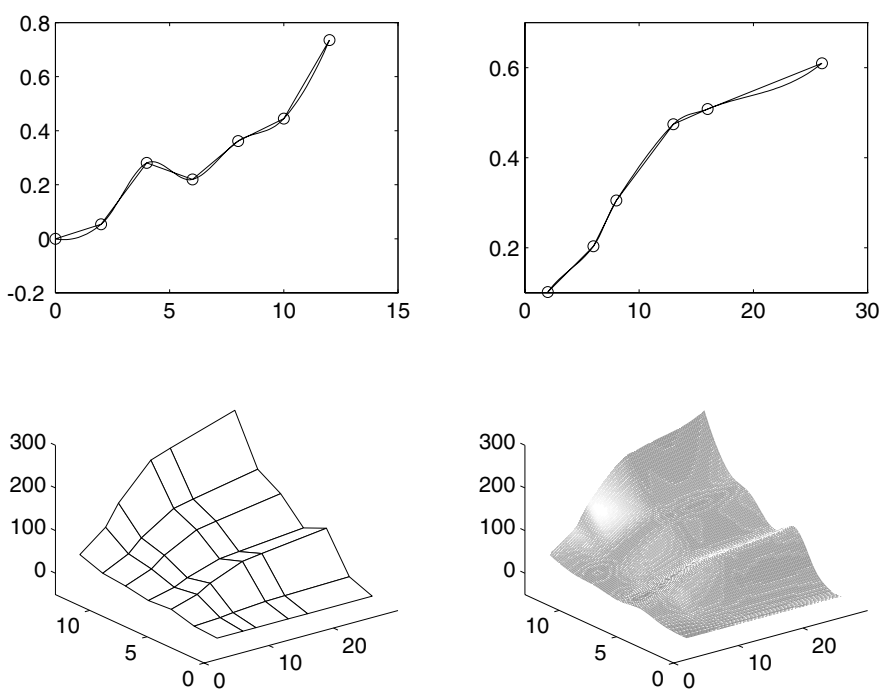

Figure 2. Top row: The singular vectors corresponding to the largest singular value and both their linear-spline and Hermite interpolants. Bottom row: at left the outer product skeleton of the singular vectors, and at right the product of the Hermite interpolating functions-a skin of the first rank-one matrix.

The resulting points were connected with linear segments yielding the bust at left in Figure 4. In spite of this coarse beginning, the resulting improvement using $1728 \times 25$ points chosen from our interpolating surface yields the Abe shown at right in Figure 4 (which we consider better in the sense that the skin looks more like the original bust than do the scanned points). The continuous, differentiable interpolation surface was
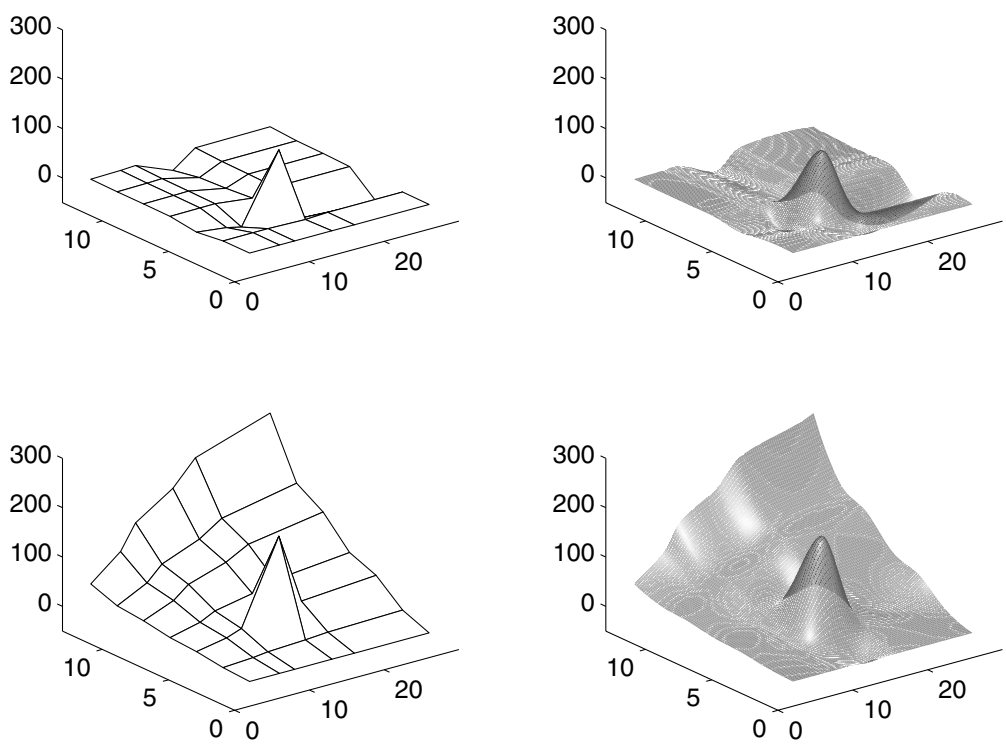

Figure 3. Top row: at left the outer product skeleton of the singular vectors corresponding to the second singular value, and at right its Hermite skin. At bottom we show the data matrix $A$ (3) and the result of adding together the rank-one interpolants to form a $C^{1}$-continuous interpolant to $A$. 


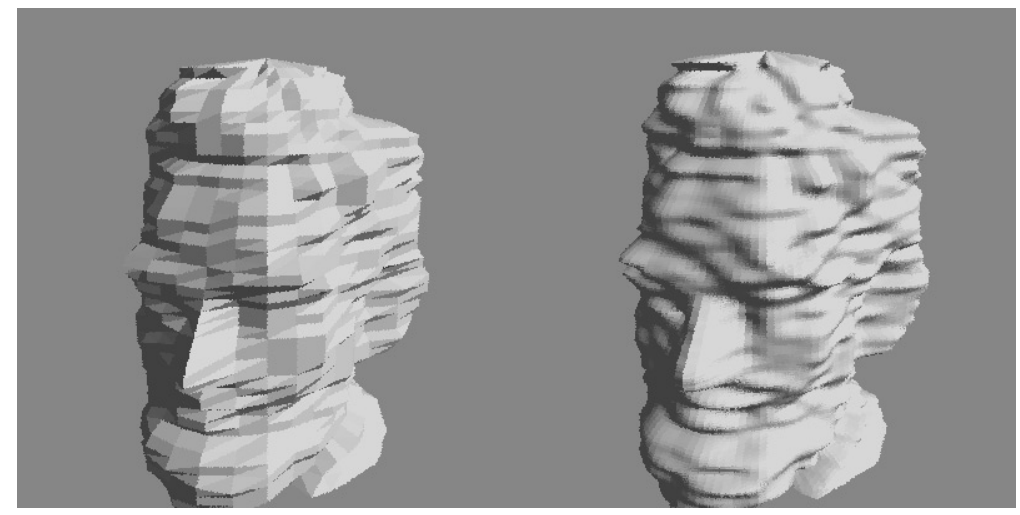

Figure 4. At left is the scanned image of Abe; at right the improved Abe, using roughly 25 times as many points obtained via Hermite interpolation.

constructed using Hermite interpolation as in Example 1. Our original scanned points were regularly spaced in the $z$ and $\theta$ coordinates of cylindrical coordinates $(r, \theta, z)$. The radial coordinates $r$ were thus a function of the $36 \times 48$ rectangular grid of $\theta$ and $z$ coordinate values, and were used as the matrix values.

\section{Conclusion.}

We have presented a simple method for the creation of surface interpolants of twodimensional data sets defined on grids. The level of differentiability desired is easily

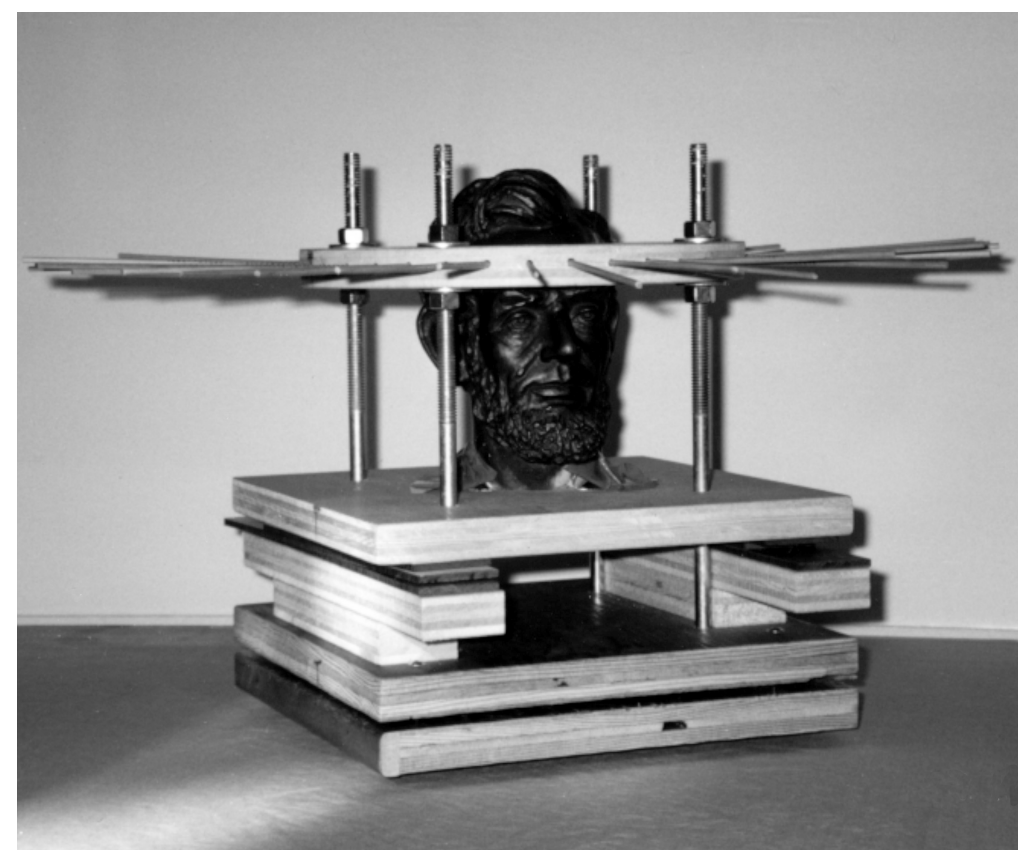

Figure 5. This crude scanner was used to read points off a bust of Abraham Lincoln, and a "mere" 1728 points required a good deal of labor! 
adjusted by choosing suitable vector interpolants, and smoothing is a readily available option (useful if the data contain noise).

While we presented a pair of examples, we have not exhausted the usefulness of SVD interpolation. In particular, we have done much work with the interpolation of parameterized surfaces (i.e., interpolating matrices of points). We are working on a higher dimensional generalization [6] (interpolating solid objects with onedimensional ones), and on the problem of interpolating "function meshes" (that is, grids of interlacing functions), the simplest case being that of the boundary curves on a rectangular region.

Acknowledgement. We wish to thank former Editor Bart Braden for his assistance in the preparation of this manuscript.

\section{References}

1. H. C. Andrews and C. Patterson, Outer product expansions and their uses in digital image processing, American Mathematical Monthly, 82:1 (1975) 1-13.

2. R. L. Burden and J. D. Faires, Numerical Analysis, 5th ed., PWS-Kent, 1993.

3. T. Hern and C. Long, Viewing some concepts and applications in linear algebra, Visualization in teaching and learning mathematics, MAA Notes Series, 19 (1991) 173-190.

4. K. Jetter and F. I. Utreras, Multivariate Approximation, World Scientific, 1992.

5. D. Kalman, A singularly valuable decomposition: the SVD of a matrix, This Journal, 27:1 (1996) 2-23.

6. A. Long, Cokriging, Kernel Estimation, and the SVD: Toward Better Geostatistical Analysis, Ph.D. thesis, Program in Applied Mathematics, University of Arizona, 1994.

7. C. Long, Visualization of matrix singular value decomposition, Mathematics Magazine, $56: 3$ (1983) 161167.

8. E. V. Shilkin and A. I. Plis, Handbook on Splines for the User, CRC Press, 1995.

9. H. Spath, Two Dimensional Spline Interpolation Algorithms, A. K. Peters, 1994.

\section{Count the Errors}

From HIP 2 B ${ }^{2}$, by Jay Darby (Hemisphere, October 2000, 116, 118, 120, 122, 147), p. 147.

"Neils Henrik Abel invented the concept of Abelian groups. These are mathematical functions that are "commutative," which basically means that changing the order of the components does not change the result. For example, multiplication and arithmetic are commutative, because $4 * 3=3 * 4$ and $3+5=5+3$."

Yes, "arithmetic" is what Mr. Darby wrote. Should not you believe such stuff could appear in print and want to check that I am not making it up you may have some difficulty. Hemisphere is the in-flight magazine of United Airlines, probably not preserved by many libraries. 